\title{
Osteoarticular Expression of Musashi-1 in an Experimental Model of Arthritis
}

\author{
Francisco O'Valle, ${ }^{1}$ Magdalena Peregrina, ${ }^{2}$ Vicente Crespo-Lora, ${ }^{1}$ \\ Pablo Galindo-Moreno, ${ }^{3}$ Maria Roman, ${ }^{4}$ Miguel Padial-Molina, ${ }^{3}$ Francisco Mesa, ${ }^{5}$ \\ Jose Aneiros-Fernandez, ${ }^{1}$ David Aguilar, ${ }^{1}$ Elena Gonzalez-Rey, ${ }^{6}$ \\ Mario Delgado, ${ }^{6}$ and Pedro Hernandez-Cortes ${ }^{7}$
}

\author{
${ }^{1}$ Department of Pathology and IBIMER, School of Medicine, University of Granada, 18012 Granada, Spain \\ ${ }^{2}$ The Spanish Institute of Social Security (INSS), 18006 Granada, Spain \\ ${ }^{3}$ Oral Surgery and Implant Dentistry Department, School of Dentistry, University of Granada, 18017 Granada, Spain \\ ${ }^{4}$ Plastic Surgery Department, Virgen de las Nieves University Hospital, 18014 Granada, Spain \\ ${ }^{5}$ Periodontics Department, School of Dentistry, University of Granada, 18017 Granada, Spain \\ ${ }^{6}$ Parasitology and Biomedicine López-Neyra Institute, CSIC, 18016 Armilla, Granada, Spain \\ ${ }^{7}$ Orthopedic Surgery Department, San Cecilio University Hospital of Granada, 18012 Granada, Spain
}

Correspondence should be addressed to Francisco O’Valle; fovalle@ugr.es

Received 23 September 2014; Revised 11 January 2015; Accepted 20 January 2015

Academic Editor: Monica Fedele

Copyright (C) 2015 Francisco O'Valle et al. This is an open access article distributed under the Creative Commons Attribution License, which permits unrestricted use, distribution, and reproduction in any medium, provided the original work is properly cited.

Background. Collagen-induced arthritis (CIA), a murine experimental disease model induced by immunization with type II collagen (CII), is used to evaluate novel therapeutic strategies for rheumatoid arthritis. Adult stem cell marker Musashi-1 (Msil) plays an important role in regulating the maintenance and differentiation of stem/precursor cells. The objectives of this investigation were to perform a morphological study of the experimental CIA model, evaluate the effect of TNF $\alpha$-blocker (etanercept) treatment, and determine the immunohistochemical expression of Msil protein. Methods. CIA was induced in 50 male DBA1/J mice for analyses of tissue and serum cytokine; clinical and morphological lesions in limbs; and immunohistochemical expression of Msil. Results. Clinically, TNF $\alpha$-blocker treatment attenuated CIA on day 32 after immunization $(P<0.001)$. Msil protein expression was significantly higher in joints damaged by CIA than in those with no lesions $(P<0.0001)$ and was related to the severity of the lesions (Spearman's rho $=0.775, P=0.0001)$. Conclusions. Treatment with etanercept attenuates osteoarticular lesions in the murine CIA model. Osteoarticular expression of Msil protein is increased in joints with CIA-induced lesion and absent in nonlesioned joints, suggesting that this protein is expressed when the lesion is produced in order to favor tissue repair.

\section{Introduction}

Collagen-induced arthritis (CIA), a murine experimental disease model induced by immunization with type II collagen (CII), shares a number of clinical, histopathological, and immunological features with rheumatoid arthritis (RA) [1]. Although its etiology is unknown, the initial stages of RA and CIA involve multiple steps that can be divided into two main phases: the initiation and establishment of autoimmunity to collagen-rich joint components, and later events associated with progressively destructive inflammatory processes [1-4].
Progression of the autoimmune response involves the development of autoreactive Thl and Th17 cells, their entry into the joint tissues, and the subsequent recruitment of inflammatory cells via multiple mediators [4]. The chronic nature of the inflammatory process in RA suggests a disturbance of immune regulation in the joint, probably caused by an excessive inflammatory response along with deficiency in the mechanisms controlling the immune response. Available therapies are based on immunosuppressive agents that inhibit the inflammatory component of RA and either reduce the relapse rate or delay disease onset. However, they have 
multiple effects, some of which are undesirable, and in the long term they do not prevent progressive clinical disability [5].

Tumor necrosis factor alpha (TNF- $\alpha$ ) is a proinflammatory cytokine expressed in the pannus of the inflamed joint in RA [6-9]. TNF $\alpha$-blocker drugs are among those currently used for the treatment of RA. The subcutaneous administration to RA patients of the TNF- $\alpha$ receptor antagonist etanercept (50 mg once a week) was found to induce clinical improvements not observed with the drugs previously used in this disease $[10,11]$. In addition, the beneficial effects of TNF $\alpha$-blocker therapy have been demonstrated by various research groups in a mouse model of CIA [12-17].

The adult stem cell marker Musashi-1 (Msil) is an RNAbinding protein of 362 amino acids with two ribonucleoprotein motifs (RBD1 and RBD2) [18] of $39 \mathrm{kDa}$ molecular weight. Msil is associated with the maintenance and asymmetric cell division of neural and epithelial progenitor cells [19]. It is expressed in various epithelial stem cells and plays an important role in regulating the maintenance and differentiation of stem/precursor cells [20, 21]. Msil is known to regulate progenitor cell function through the posttranscriptional regulation of its target RNA [20]. Msil also acts as an important positive regulator of cell proliferation and inhibitor of apoptosis by reducing Notch-1 expression [22].

Modulation of Msil immunohistochemical expression has been identified in a murine model of inflammatory colitis [23], and it has been speculated that Msil might promote cell proliferation by accelerating the cell cycle in neoplastic cells [24], suggesting a role for this protein in tissue repair in different processes and as a potential therapeutic target in regenerative medicine.

The objectives of this study were to morphologically analyze the experimental collagen-induced arthritis model, to evaluate the effect of treatment with a TNF $\alpha$-blocker (etanercept), and to determine the immunohistochemical expression of Msil protein.

\section{Material and Methods}

2.1. Induction and Treatment of Collagen-Induced Arthritis (CIA). CIA was induced in 50 male DBA1/J mice (7 to 10 weeks old; Jackson Laboratories, Bar Harbor, ME) by subcutaneous injection into the tail base with $200 \mu \mathrm{g}$ bovine CII (Sigma, St. Louis, MO) at day 0 and with $100 \mu \mathrm{g}$ CII at day 21, both emulsified in complete Freund's adjuvant containing $200 \mu \mathrm{g}$ Mycobacterium tuberculosis H37 RA (Difco, Detroit, Michigan). Treatment consisted of the intraperitoneal injection of $2 \mathrm{mg}$ of etanercept (Enbrel, Wyeth Europa Ltd., UK; E-group) or of phosphate-buffered saline (PBS; untreated control, C-group) once a week for four weeks (on days 25, 32,39 , and 46) starting at day 25 after immunization, when all mice showed established arthritis (clinical score $>2$ ). Mice were evaluated by two independent blinded examiners every other day and monitored for signs of arthritis onset according to the following clinical score: grade 0 , no swelling; grade 1, slight swelling and erythema; grade 2, moderate swelling and edema; grade 3, extreme swelling and pronounced edema; or grade 4, joint rigidity. Each limb was graded, giving a maximum possible score of 16 per animal. Paw swelling was assessed by measuring the thickness of the affected hind paws with 0 to $10 \mathrm{~mm}$ calipers (on days 25, 32, 39, 46, and 53). All experiments were performed in a European Union-certified laboratory following national guidelines for the ethical care of animals (RD 53/2013, EU Directive 63/2010).

2.2. Cytokine Determination. Protein extracts were isolated by homogenization of joints ( $50 \mathrm{mg}$ tissue/mL) in $50 \mathrm{mM}$ Tris- $\mathrm{HCl}, \mathrm{pH} 7.4$, with $0.5 \mathrm{mM}$ DTT and proteinase inhibitor cocktail $(10 \mu \mathrm{g} / \mathrm{mL}$, Sigma) for cytokine determination in joints, and serum samples were collected at the disease peak (day 40); serum and joint cytokine and chemokine levels were determined by specific sandwich ELISAs using capture/ biotinylated detection Abs from BD Pharmingen (San Diego, CA) according to the manufacturer's recommendations.

2.3. Histopathological Study. For the histopathological study, mice were anesthetized with ether and sacrificed by cervical dislocation at day $15(n=20), 21(n=20)$, or $28(n=10)$ after commencement of treatment with etanercept or PBS (i.e., days 39, 46, and 53 after immunization). They were fixed in $10 \%$ buffered formalin for 24 hours, decalcified with Decalcifier I, containing formaldehyde $(10 \% \mathrm{w} / \mathrm{v})$, formic acid $(8 \%$ $\mathrm{w} / \mathrm{v})$, and methanol $(1 \% \mathrm{w} / \mathrm{v})$ (Surgipath softener I Europe Ltd., Peterborough, UK) for $24 \mathrm{~h}$ in oven at $37^{\circ} \mathrm{C}$. Next, the four limbs were sectioned longitudinally, dehydrated with alcohol, and embedded in paraffin in an automatic tissue processor Excelsior ES (Thermo Scientific, CA, USA); $4 \mu \mathrm{m}$ sections were stained with hematoxylin and eosin (H\&E) or Masson trichrome stain. Histopathological changes were scored in a blinded manner based on cell infiltration, cartilage destruction, and bone erosion parameters as previously described [25]. Cell infiltration was scored on a scale of $0-$ 3 according to the number of affected joints ( 0 : none, $1:<2,2$ : $3-5 ; 3$ : $>5$ joints), and the amount of inflammatory cells in the synovial cavity (exudate) and synovial tissue (infiltrate) was also recorded. Cartilage destruction was graded on a scale of $0-3$, ranging from the appearance of dead chondrocyte (empty lacunae) to complete loss of articular cartilage. Bone erosions were graded on a scale of $0-3$, ranging from normal bone appearance to fully eroded cortical bone structure in patella and femur condyle. Pannus and involvement of the bone marrow and/or soft tissue were treated as dichotomous variables (present or absent).

2.4. Immunohistochemical Analysis. Decalcified and paraffinembedded sections were dewaxed, hydrated, and heat-treated in $1 \mathrm{mM}$ EDTA $\mathrm{pH} 8$ in an antigen retrieval PT module (Thermo Fisher Scientific Inc., Waltham, MA) at $95^{\circ} \mathrm{C}$ for $20 \mathrm{~min}$. Sections were incubated for $16 \mathrm{~h}$ at $4^{\circ} \mathrm{C}$ with the prediluted polyclonal antibody against Musashi-1 (SigmaAldrich, Barcelona, Spain) at 1:100 dilution to identify cellular expression. An automatic immunostainer (Autostainer 480, Thermo Fisher Scientific Inc.) was used for the immunohistochemical study, applying the peroxidase conjugated micropolymer method and developing with diaminobenzidine (Ultravision Quanto, Master Diagnóstica, Granada, Spain). Expression was assessed semiquantitatively on a scale 
TABLE 1: Comparative study of morphological variables in DBA1/J mice after 21 and 28 days of starting treatment.

\begin{tabular}{|c|c|c|c|c|c|c|}
\hline \multirow{2}{*}{ Variables } & Control group & Etanercept group* & $P$ values $^{\dagger}$ & Control group & Etanercept group* & $P$ values $^{\dagger}$ \\
\hline & \multicolumn{2}{|c|}{ Day 46 after immunization } & \multicolumn{4}{|c|}{ Day 53 after immunization } \\
\hline Number of joints & $2.1 \pm 0.69$ & $1.8 \pm 0.88$ & 0.412 & $2.45 \pm 1.01$ & $1.9 \pm 0.56$ & 0.006 \\
\hline Pannus & $1 \pm 0.21$ & $0.9 \pm 0.35$ & 0.449 & $0.7 \pm 0.31$ & $1 \pm 0$ & 0.467 \\
\hline $\mathrm{B} \& \mathrm{C}$ injury & $1.8 \pm 0.71$ & $1.3 \pm 0.78$ & 0.278 & $2 \pm 0.94$ & $1.55 \pm 0.68$ & 0.045 \\
\hline Inflammation & $1.5 \pm 1$ & $1.3 \pm 1$ & 0.661 & $1.55 \pm 0.83$ & $1.1 \pm 0.69$ & 0.036 \\
\hline Bone marrow & $0.6 \pm 0.47$ & $0.5 \pm 0.39$ & 0.613 & $0.55 \pm 0.42$ & $0.55 \pm 0.43$ & 0.930 \\
\hline Soft tissue & $0.15 \pm 0.15$ & $0.2 \pm 0.21$ & 0.557 & $0.35 \pm 0.31$ & $0.15 \pm 0.15$ & 0.010 \\
\hline Mean score & $6.85 \pm 2.95$ & $5.8 \pm 3.37$ & 0.469 & $8 \pm 3.55$ & $6 \pm 2.12$ & 0.011 \\
\hline
\end{tabular}

Values are expressed as mean \pm standard deviation; ${ }^{*}$ treatment: etanercept $2 \mathrm{mg} /$ week; B \& C: bone and cartilage; ${ }^{\dagger}$ Student's $t$-test. See Figure 3 for details.

of 0 to 3 ( 0 : absence, 1 : mild [ $<10 \%$ positive cells], 2 : moderate [10 to $25 \%], 3$ : intense [>25\%] in bone tissue, hyaline cartilage, joint capsule, synovium, ligaments, striated muscle cells, endothelial cells, and adipocytes). The variables were subsequently categorized in two groups (presence/absence of osteoarticular lesion), calculating the total Msil expression score for each group.

2.5. Statistical Analysis. SPSS 20.0 (IBM Inc., Chicago, IL) was used for the statistical analysis. The normality of the distribution of variables was examined with the one-dimensional Kolmogorov-Smirnov test. Results were expressed as mean \pm standard deviation for normally distributed continuous variables and frequencies for categorical variables. The bivariate tests and Spearman correlation coefficient used are reported in the table footnotes. A $P$ value of 0.05 was accepted as the statistical significance threshold.

\section{Results}

The clinical score was significantly attenuated in mice with CIA after 1 week of systemic treatment with the TNF $\alpha$ blocker etanercept $(P<0.001)$ in comparison to untreated mice (Figure 1). This treatment also significantly reduced serum and joint tissue levels of Th1-mediated proinflammatory cytokines and increased levels of the anti-inflammatory cytokine IL10 (Figure 2).

The histopathological study demonstrated a CIA induction efficiency of $95 \%$ in DBA1/J mice, which showed pannus formation and chronic lymphocytic/monocytic inflammatory infiltrate with acute phenomena (neutrophil leukocytes) that involved the joint soft tissues (Figure 3). These mice also showed the presence of necrotic cells in the intraarticular space, secondary destruction of the joint cartilage, and increased destruction of bone tissue through osteoclastic activation, with sporadic involvement of the bone medulla and extension of inflammatory infiltrate into periarticular soft tissues (Figure 3). Injection of etanercept in CIA mice moderately decreased most of the histopathological signs of arthritis, especially after four weeks of treatment (day 53 after immunization, Table 1). No significant differences in morphologic variables were observed between untreated and etanercept-treated mice after three weeks of treatment (day 46 after immunization, Table 1); however, stratification

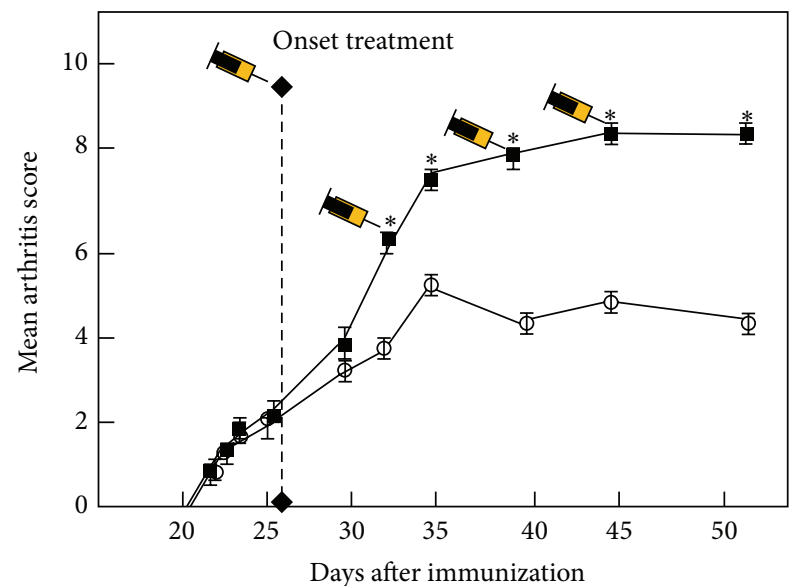

Figure 1: TNF $\alpha$-blocker administration attenuates clinical CIA. Clinical scores in mice with CIA intraperitoneally treated from day 25 after immunization with PBS (control, C-group, closed squares) or TNF $\alpha$-blocker ( $2 \mathrm{mg} /$ week, E-group, open circles) once per week for four weeks. Values are the mean \pm SD of 20 mice per group. Differences were significant at $P<0.001$ (asterisks) for E-group versus C-group at indicated time points.

of the histopathological results according to the absence or presence of lesions $(0=$ absence, $1=$ presence $)$ showed that the etanercept treatment attenuated the progression of clinical inflammation in the limbs (mean of 0.55 in the etanercept group versus 0.85 in control group after 21 days of treatment; $P<0.003$, Student's $t$-test).

The immunohistochemical expression of Msil was then investigated in different tissue components in the joints of untreated and etanercept-treated CIA mice. Msil-specific staining was mainly observed at nuclear level in chondrocytes and spindle-shaped mesenchymal cells of the articular capsule and ligaments (Figure 4). Scant expression was detected in osteocytes, synoviocytes, or inflammatory cells in articular or periarticular lesions or in medullary bone tissue (Figure 4). No Msil expression was observed in striated muscle cells or mature adipocytes (Figure 4 ).

Quantitative analysis of Msil expression in the different groups revealed significantly higher expression in joints with CIA-induced articular lesions than in joints without lesions $(P<0.0001$, Student's $t$-test, Figure 4$)$. Significant positive 


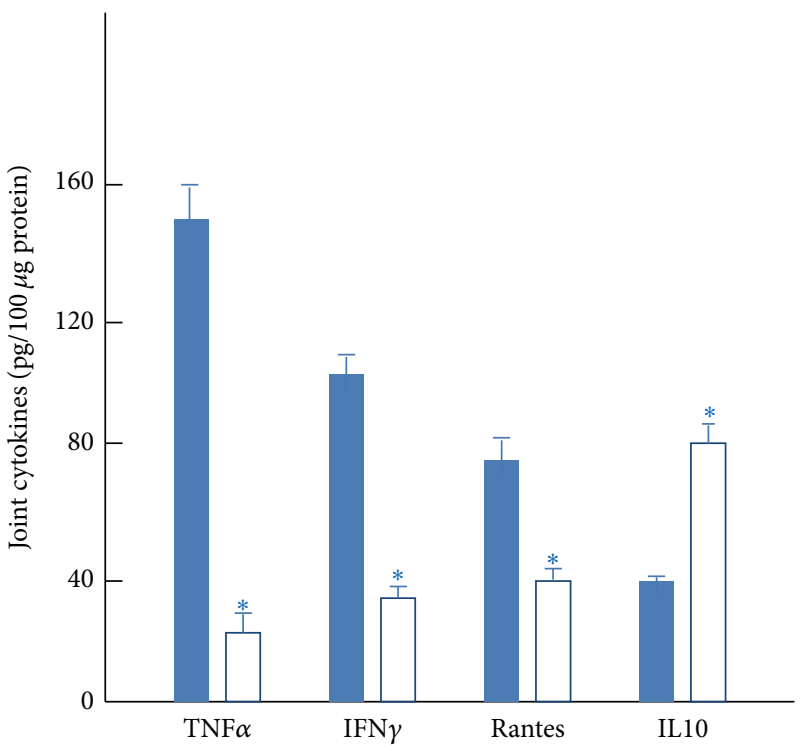

(a)

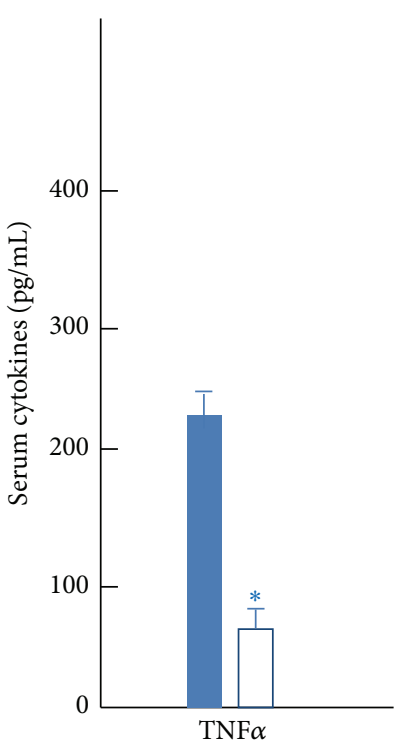

(b)

Figure 2: TNF $\alpha$-blocker administration decreases inflammatory response in CIA. DBA1/J mice with established CIA (day 25 after immunization) were intraperitoneally injected with PBS (closed columns) or anti-TNF $\alpha$ ( $2 \mathrm{mg} / \mathrm{week}$, open columns) once per week. Systemic and local expression of inflammatory mediators was assayed in protein extracts from joints of hind limbs (a) and sera (b) isolated at day 40 after immunization; $n=3$ to 4 mice/group. ${ }^{*} P<0.01$ versus controls.

TABLE 2: Spearman's correlation coefficient (rho) for Msil immunohistochemical expression between different articular components.

\begin{tabular}{lcccc}
\hline & Cartilage & Capsule & Ligament & Synovium \\
\hline Cartilage & 1 & $0.225^{*}$ & $0.308^{* *}$ & $0.556^{* *}$ \\
Capsule & & 1 & $0.663^{* *}$ & $0.389^{* *}$ \\
Ligament & & & 1 & $0.260^{*}$ \\
Synovium & & & & 1 \\
\hline
\end{tabular}

${ }^{*}$ Significant correlation at 0.05 (bilateral); ${ }^{* *}$ significant correlation at 0.01 (bilateral).

correlations were also observed between the presence of osteoarticular lesion and Msil expression (Spearman's rho: $0.775, P=0.001$ ) and among Msil expression levels at the different sites (capsule, ligament, synovium, and cartilage) (Table 2). However, although a slight decrease in Msil expression was observed in affected joints in etanercept-treated CIA mice, no statistically significant differences in articular Msil expression were found between PBS-treated and etanercepttreated CIA mice after three $(P=0.449$, Student's $t$-test $)$ or four $(P=0.080$, Student's $t$-test $)$ weeks of treatment (Figure 5).

\section{Discussion}

This study confirmed that TNF $\alpha$-blocker treatment attenuates CIA-induced histopathological lesions in the joints of CIA-susceptible DBA1/J mice and revealed, for the first time, a more intense expression of Msil protein in cartilage, ligament, articular capsule, mesenchymal cells, and osteocytes in CIA-lesioned versus nonlesioned joints.
Although no animal model of RA completely replicates the human disease, the CIA model employed in this study has been widely used for the testing and development of RA therapies [26-29]. In fact, the CIA induction rate was very high (>95\%) in the present study. Likewise, DBA1/J mice were selected rather than other animals (e.g., rats) [30] because better outcomes are obtained in genetically modified strains and the arthritis is more similar to human RA [31, 32]. Major insights into the molecular mechanisms of inflammatory arthritis recently emerged from the study of murine models of RA-like disease using genetically deficient or transgenic mice or a combined murine model $\left(\mathrm{K} / \mathrm{BxA}^{\mathrm{g} 7}\right)$ that spontaneously develops both RA-like disease and atherosclerosis [33]. However, these studies may be limited by the differences between human and murine immune systems. Current efforts to develop an animal model that utilizes human immune cells will allow study of their function in the initiation and propagation of inflammatory arthritis [34].

RA is a chronic debilitating disease in which the induction of autoimmunity to collagen-rich joint components underlies the onset of the disease and the subsequent destructive inflammatory process. Progression of the autoimmune response implies the development of autoreactive Th1 (producing IFN $\gamma$ and TNF $\alpha$ ) and Th17 (producing Th17) cells, their entry into articular tissue, and the release of proinflammatory cytokines and chemokines, which promote macrophage and neutrophil infiltration and activation $[3,35$, 36]. Excessive production by infiltrating inflammatory cells of inflammatory cytokines, free radicals, and extracellular matrix-degrading enzymes plays a critical role in cartilage damage and bone erosion. A desirable therapeutic approach would be to prevent the activation of inflammatory and 


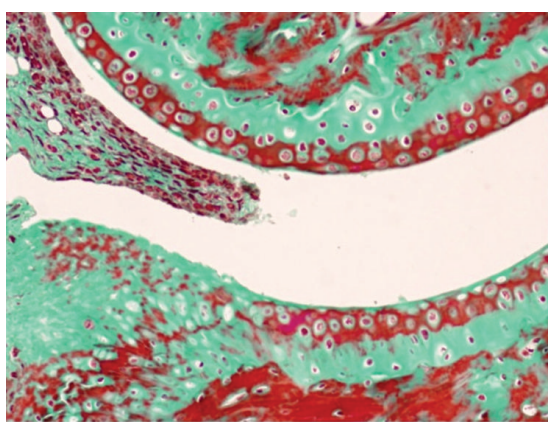

(A)

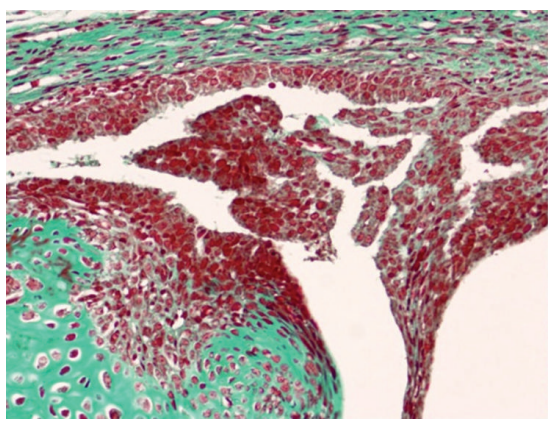

(C)

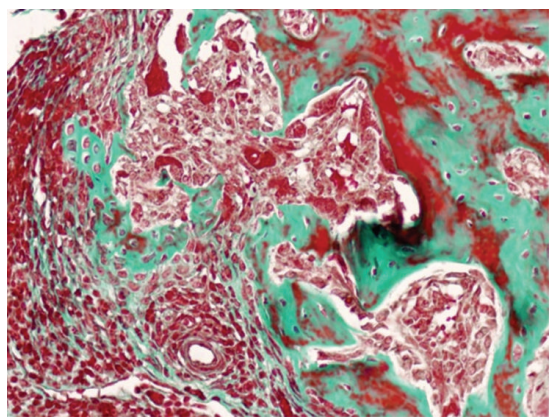

(E)

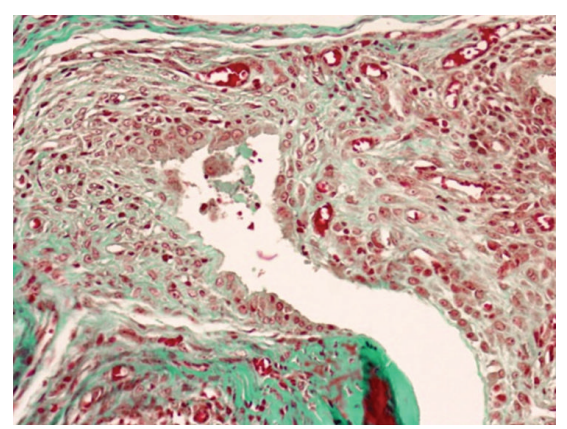

(B)

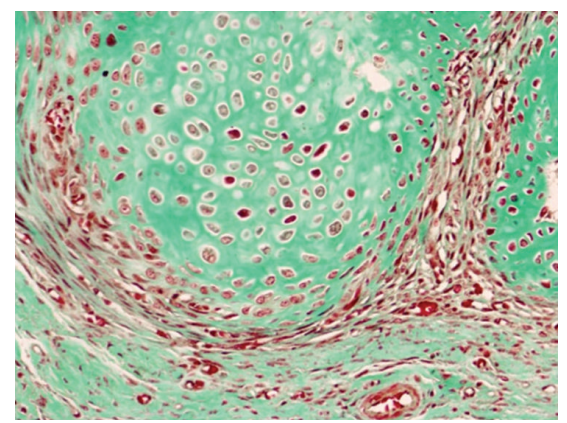

(D)

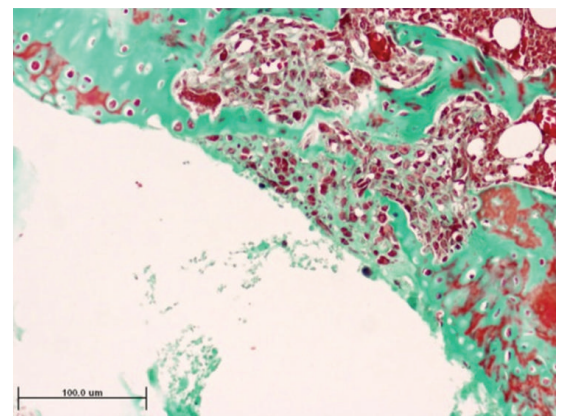

(F)

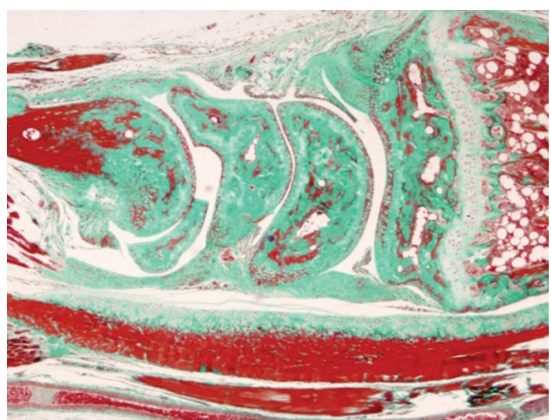

(A)

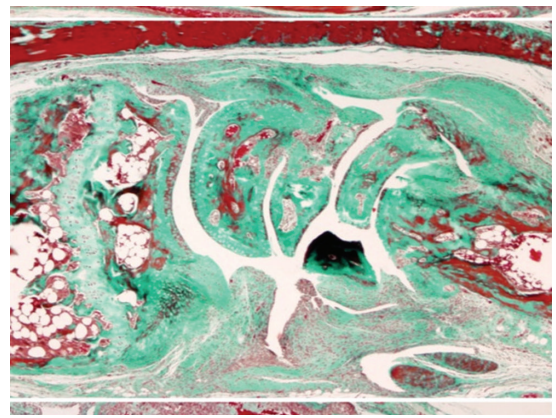

(B)

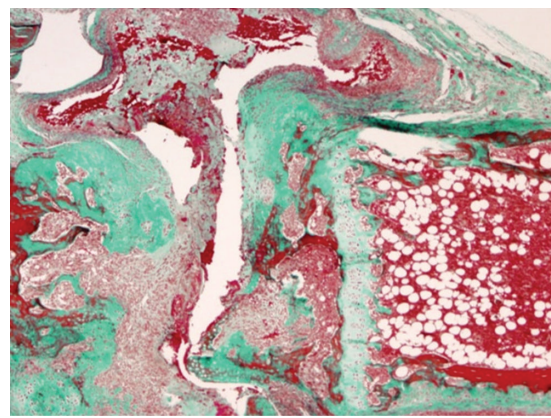

(C)

(b)

(a)

Figure 3: Morphological features of articular lesion in CIA model in DBA1/J mice. (a): (A) Unaffected joint. (B) Pannus. (C) Synovial hyperplasia with chronic inflammatory infiltrate. (D) Hyaline cartilage surrounded by spindle-shaped mesenchymal cells. (E) Bone destruction mediated by osteoclast activation. (F) Partial destruction of articular cartilage. Bar $100 \mu \mathrm{m}$ (Masson's trichrome, original magnification $\times 20$ ). (b): (A) Limb without joint lesions. (B) Partial response to etanercept with persistence of pannus (E-group). (C) Intense joint lesion with pannus and chronic inflammatory infiltrate in articular cavity and partial destruction of bone tissue (C-group) (Masson's trichrome, original magnification $\times 4$ ).

autoimmune components. Our group previously demonstrated that 5-aminoisoquinolinone, a poly(ADP-ribose) polymerase-1 inhibitor, significantly reduces the incidence and severity of established CIA, completely abrogating joint swelling and cartilage/bone destruction by downregulating inflammation and the Thl response [37]. The administration of TNF $\alpha$-blocker to arthritic mice decreases the CII-specific Th1-mediated cytokine response through direct action on the synovium [38-40]. The present results confirm that treatment of established CIA with etanercept reduces articular levels of Thl cytokines (IFN $\gamma$ and TNF $\alpha$ ) and inflammatory chemokines. This effect may be directly related to a decrease in inflammatory infiltrates in the joints of etanercept-treated mice. However, the fact that levels of the anti-inflammatory cytokine IL10 were increased by the injection of etanercept supports the proposition that this TNF $\alpha$-blocker also promotes a bias towards a regulatory/anti-inflammatory response.

Etanercept and other blockers of TNF $\alpha$ action (infliximab, adalimumab, golimumab, and certolizumab pegol) offer specific anti-cytokine therapies but induce a general immunosuppressive action. Etanercept has become the drug of choice for late stage RA with reasonable safety, and the present study confirms that it attenuates the progression of histopathological lesions, as demonstrated in CIA murine models [12-17] and in the clinical setting [41, 42]. However, 


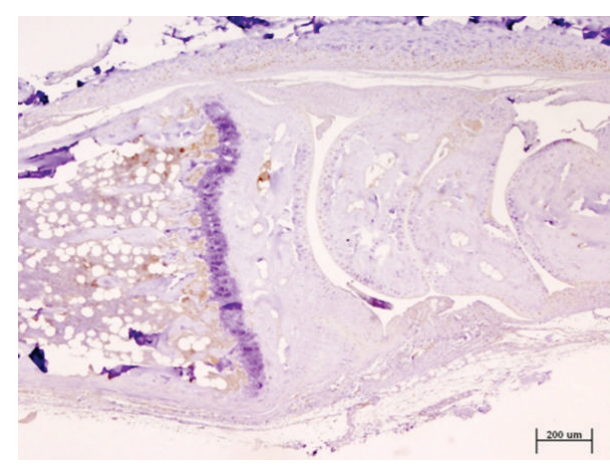

(A)

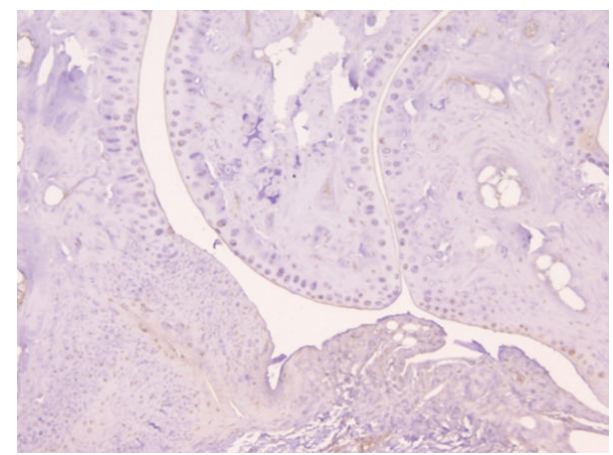

(A)

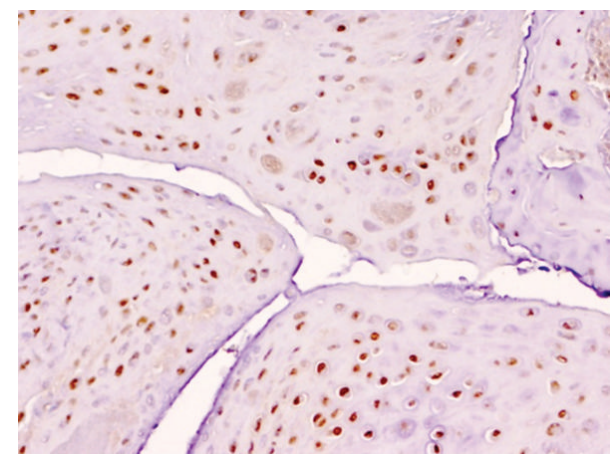

(C)

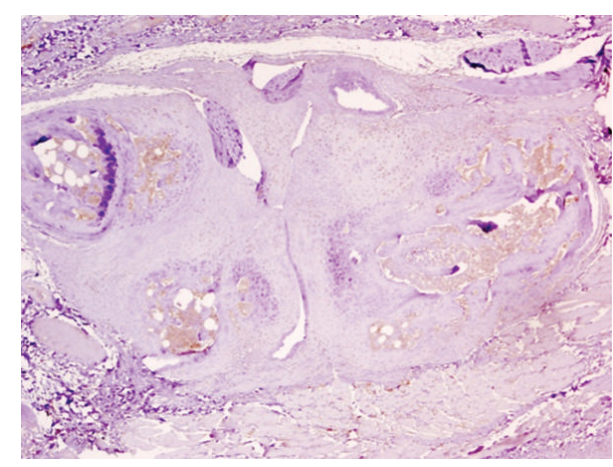

(B)

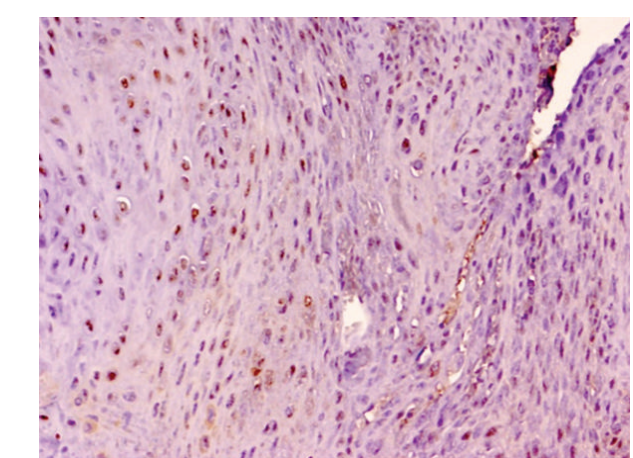

(B)

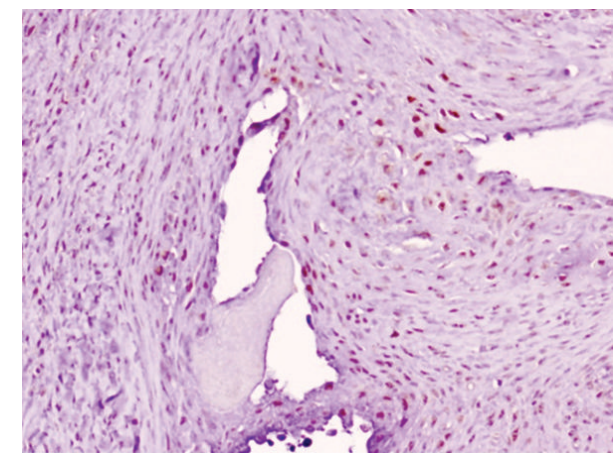

(D)

(b)

Figure 4: Immunohistochemical expression of Musashi-1 (Msil) in CIA model in DBA1/J mice. (a): (A) Scant Msil expression was detected in chondrocytes, synoviocytes, and inflammatory cells in articular or periarticular lesions or in medullary bone tissue in joints with no morphological changes. (B) Nuclear expression of Msil in mesenchymal cells, articular capsule, and articular cartilage. Bar $200 \mu \mathrm{m}$ (micropolymer peroxidase-based method, original magnification $\times 4$ ). (b): (A) Very scant nuclear Msil expression in joint with no morphological changes (E-group). (B), (C), and (D) Moderate Msil expression in mesenchymal cells, articular capsule, and articular cartilage in CIA-lesioned joints (C-group) (micropolymer peroxidase-based method, original magnification $\times 20$ ).

etanercept must be taken frequently, it is expensive, and it increases the susceptibility of the patient to infections [43].

The present study provides the first report on the osteoarticular expression of Msil. No direct evidence has been published to date linking Msil with osteoarticular regeneration. Msil is involved in the regulation of self-renewal of stem cells. In order to maintain their unlimited capacity to divide, stem cells require controlled temporal and spatial protein expression. The Musashi family of RNA-binding proteins exerts this essential translational control (via repression and activation) in order to regulate multiple stem cell populations [44], and Msil-dependent posttranscriptional enhancement of m-Numb is crucial in epithelial regeneration [45]. Nuclear expression of Msil was more intense in the presence of osteoarticular lesion and was not observed in nonlesioned joints, with or without anti-TNF $\alpha$ treatment. In previous studies on fractures in rats, we observed a marked increase in Msil expression in the reparative fibrocartilaginous tissue of the fracture callus (data not shown). Msil protein regulates the transcription and differentiation of mesenchymal stem 


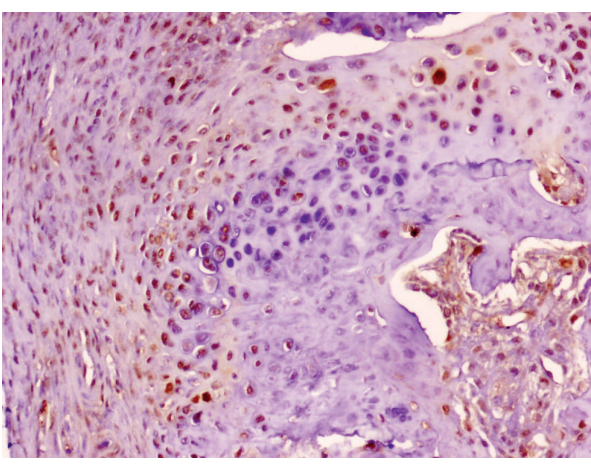

(a)

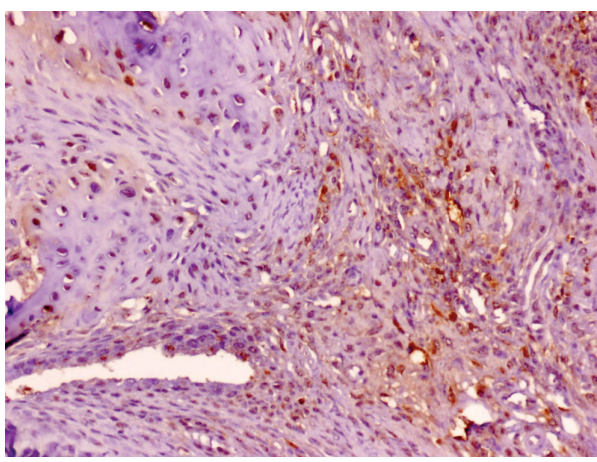

(c)

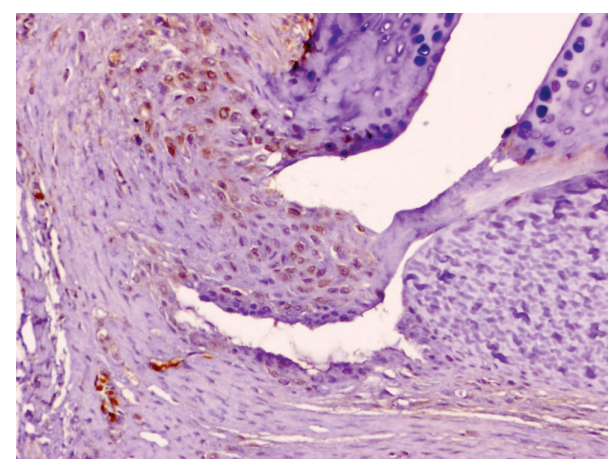

(b)

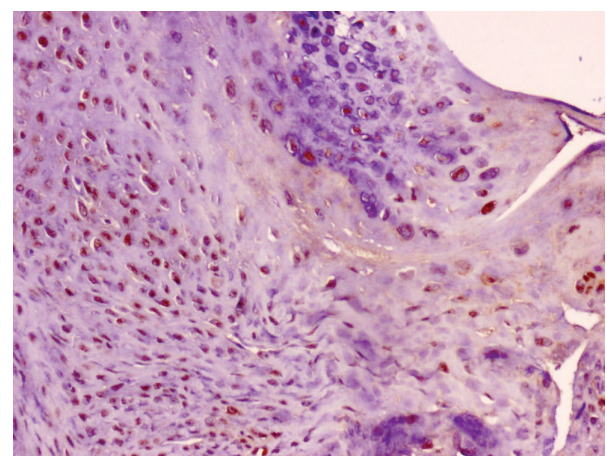

(d)

FIGURE 5: Representative immunohistochemical expression of Msil in E-group versus C-group in CIA model (DBA1/J mice). (a) Moderate Msil expression in mesenchymal cells, articular capsule, and articular cartilage in CIA-lesioned joints at 21 days after treatment (E-group); (b) moderate Msil expression in CIA-lesioned joints at 21 days after treatment (C-group); (c) joint Msil expression at 28 days after treatment (E-group); (d) joint Msil expression at 28 days after treatment (C-group) (micropolymer peroxidase-based method, original magnification $\times 20)$.

cells; therefore, its presence in these tissues suggests its involvement in tissue repair and regeneration processes.

Our novel findings on the expression of Msil in osteoarticular tissues may support the participation of this protein in tissue regeneration processes and suggest the involvement of adult mesenchymal stem cells in the repair of these tissues. These data may serve as a basis for future investigations on repair processes in cartilaginous and bone tissues.

\section{Conclusions}

Treatment with etanercept attenuates the osteoarticular lesions in the murine model of CIA. The osteoarticular expression of Msil protein is increased in joints with CIA-induced lesion and absent in nonlesioned joints, suggesting that this protein is expressed when the lesions are produced in order to favor tissue repair.
Abbreviations
AIQ: 5-Aminoisoquinolinone
CIA: Collagen-induced arthritis
CII: Type II collagen
IFN $\gamma$ : Gamma interferon
Msil: Musashi-1

RA: Rheumatoid arthritis

TNF $\alpha$ : Tumor necrosis factor alpha.

\section{Conflict of Interests}

The authors do not have any financial interests, either directly or indirectly, in the products or information listed in the paper.

\section{Authors' Contribution}

Francisco O'Valle, Pedro Hernandez-Cortes, Elena Gonzalez-Rey, and Mario Delgado conceived, designed, and performed the mouse model, coordinated in the study, and drafted the paper. Magdalena Peregrina, Pablo GalindoMoreno, Maria Roman, Miguel Padial-Molina, and Francisco Mesa analyzed the clinical and biochemical data and drafted the paper. Vicente Crespo, Jose Aneiros Fernandez, and David Aguilar analyzed the morphology and immunohistochemical data. All authors read and approved the final version of this paper.

\section{Acknowledgments}

The authors thank M.D. Rodríguez-Martínez, from the Pathology Department of the School of Medicine, University of 
Granada, for her expert technical assistance and R. Davies, professional translator, for help with the English version of the paper. This investigation was partially supported by Research Group \#CTS-138 (Junta de Andalucía, Spain).

\section{References}

[1] D. D. Anthony and T. M. Haqqi, "Collagen-induced arthritis in mice: an animal model to study the pathogenesis of rheumatoid arthritis," Clinical and Experimental Rheumatology, vol. 17, no. 2, pp. 240-244, 1999.

[2] M. Feldmann, F. M. Brennan, and R. N. Maini, "Role of cytokines in rheumatoid arthritis," Annual Review of Immunology, vol. 14, pp. 397-440, 1996.

[3] D. D. Brand, A. H. Kang, and E. F. Rosloniec, "Immunopathogenesis of collagen arthritis," Springer Seminars in Immunopathology, vol. 25, no. 1, pp. 3-18, 2003.

[4] E. Gonzalez-Rey, A. Chorny, F. O’Valle, and M. Delgado, "Adrenomedullin protects from experimental arthritis by down-regulating inflammation and Thl response and inducing regulatory T cells," The American Journal of Pathology, vol. 170, no. 1, pp. 263-271, 2007.

[5] M. Feldmann and R. N. Maini, "Anti-TNFalpha therapy of rheumatoid arthritis: what have we learned?" Annual Review of Immunology, vol. 19, pp. 163-196, 2001.

[6] J. C. Beckham, D. S. Caldwell, B. L. Peterson et al., "Disease severity in rheumatoid arthritis: relationships of plasma tumor necrosis factor- $\alpha$, soluble interleukin 2-receptor, soluble CD4/CD8 ratio, neopterin, and fibrin D-dimer to traditional severity and functional measures," Journal of Clinical Immunology, vol. 12, no. 5, pp. 353-361, 1992.

[7] F. M. Brennan, D. Chantry, A. Jackson, R. Maini, and M. Feldmann, "Inhibitory effect of TNF $\alpha$ antibodies on synovial cell interleukin-1 production in rheumatoid arthritis," The Lancet, vol. 2, no. 8657, pp. 244-247, 1989.

[8] X. Sáez-Llorens, H. S. Jafari, K. D. Olsen, H. Nariuchi, E. J. Hansen, and G. H. McCracken Jr., "Induction of suppurative arthritis in rabbits by Haemophilus endotoxin, tumor necrosis factor- $\alpha$, and interleukin- $1 \beta$," Journal of Infectious Diseases, vol. 163, no. 6, pp. 1267-1272, 1991.

[9] D. Tracey, L. Klareskog, E. H. Sasso, J. G. Salfeld, and P. P. Tak, "Tumor necrosis factor antagonist mechanisms of action: a comprehensive review," Pharmacology \& Therapeutics, vol. 117, no. 2, pp. 244-279, 2008.

[10] S. Dhillon, K. A. Lyseng-Williamson, and L. J. Scott, "Etanercept: a review of its use in the management of rheumatoid arthritis," Drugs, vol. 67, no. 8, pp. 1211-1241, 2007.

[11] B. Haraoui and V. Bykerk, "Etanercept in the treatment of rheumatoid arthritis," Therapeutics and Clinical Risk Management, vol. 3, no. 1, pp. 99-105, 2007.

[12] G. J. Thorbecke, R. Shah, C. H. Leu, A. P. Kuruvilla, A. M. Hardison, and M. A. Palladino, "Involvement of endogenous tumor necrosis factor $\alpha$ and transforming growth factor $\beta$ during induction of collagen type II arthritis in mice," Proceedings of the National Academy of Sciences of the United States of America, vol. 89, no. 16, pp. 7375-7379, 1992.

[13] R. O. Williams, M. Feldmann, and R. N. Maini, "Anti-tumor necrosis factor ameliorates joint disease in murine collageninduced arthritis," Proceedings of the National Academy of Sciences of the United States of America, vol. 89, no. 20, pp. 97849788, 1992.
[14] P. F. Piguet, G. E. Grau, C. Vesin, H. Loetscher, R. Gentz, and W. Lesslauer, "Evolution of collagen arthritis in mice is arrested by treatment with anti-tumour necrosis factor (TNF) antibody or a recombinant soluble TNF receptor," Immunology, vol. 77, no. 4, pp. 510-514, 1992.

[15] P. H. Wooley, J. Dutcher, M. B. Widmer, and S. Gillis, "Influence of a recombinant human soluble tumor necrosis factor receptor FC fusion protein on type II collagen-induced arthritis in mice," Journal of Immunology, vol. 151, no. 11, pp. 6602-6607, 1993.

[16] R. O. Williams, J. Ghrayeb, M. Feldmann, and R. N. Maini, "Successful therapy of collagen-induced arthritis with TNF receptor-IgG fusion protein and combination with anti-CD4," Immunology, vol. 84, no. 3, pp. 433-439, 1995.

[17] Q. T. Wang, Y. J. Wu, B. Huang et al., "Etanercept attenuates collagen-induced arthritis by modulating the association between BAFFR expression and the production of splenic memory B cells," Pharmacological Research, vol. 68, no. 1, pp. 38-45, 2013.

[18] T. Nagata, R. Kanno, Y. Kurihara et al., "Structure, backbone dynamics and interactions with RNA of the C-terminal RNAbinding domain of a mouse neural RNA-binding protein, Musashil," The Journal of Molecular Biology, vol. 287, no. 2, pp. 315-330, 1999.

[19] H. Okano, H. Kawahara, M. Toriya, K. Nakao, S. Shibata, and T. Imai, "Function of RNA-binding protein Musashi-1 in stem cells," Experimental Cell Research, vol. 306, no. 2, pp. 349-356, 2005.

[20] H. Okano, T. Imai, and M. Okabe, "Musashi: a translational regulator of cell fate," Journal of Cell Science, vol. 115, no. 7, pp. 1355-1359, 2002.

[21] Y. Kaneko, S. Sakakibara, T. Imai et al., "Musashil: an evolutionally conserved marker for CNS progenitor cells including neural stem cells," Developmental Neuroscience, vol. 22, no. 1-2, pp. 139153, 2000.

[22] S. M. Sureban, R. May, R. J. George et al., "Knockdown of RNA binding protein musashi-1 leads to tumor regression in vivo," Gastroenterology, vol. 134, no. 5, pp. 1448-1458, 2008.

[23] T. Fukui, H. Takeda, H.-J. Shu et al., "Investigation of Musashi-1 expressing cells in the murine model of dextran sodium sulfateinduced colitis," Digestive Diseases and Sciences, vol. 51, no. 7, pp. 1260-1268, 2006.

[24] X. Liu, W. T. Yang, and P. S. Zheng, "Msil promotes tumor growth and cell proliferation by targeting cell cycle checkpoint proteins p21, p27 and p53 in cervical carcinomas," Oncotarget, vol. 5, no. 21, pp. 10870-10885, 2014.

[25] M. Delgado, C. Abad, C. Martinez, J. Leceta, and R. P. Gomariz, "Vasoactive intestinal peptide prevents experimental arthritis by downregulating both autoimmune and inflammatory components of the disease," Nature Medicine, vol. 7, no. 5, pp. 563$568,2001$.

[26] K. Kannan, R. A. Ortmann, and D. Kimpel, "Animal models of rheumatoid arthritis and their relevance to human disease," Pathophysiology, vol. 12, no. 3, pp. 167-181, 2005.

[27] Y. G. Cho, M. L. Cho, S. Y. Min, and H. Y. Kim, “Type II collagen autoimmunity in a mouse model of human rheumatoid arthritis," Autoimmunity Reviews, vol. 7, no. 1, pp. 65-70, 2007.

[28] R. Holmdahl, L. Jansson, E. Larsson, K. Rubin, and L. Klareskog, "Homologous type II collagen induces chronic and progressive arthritis in mice," Arthritis \& Rheumatism, vol. 29, no. 1, pp. 106113, 1986. 
[29] A. M. Malfait, R. O. Williams, A. S. Malik, R. N. Maimi, and M. Feldman, "Chronic relapsing homologous collageninduced arthritis in DBA/1 mice as a model for testing diseasemodifying and remission-inducing therapies," Arthritis \& Rheumatism, vol. 44, no. 5, pp. 1215-1224, 2001.

[30] J. M. Stuart, M. A. Cremer, A. S. Townes, and A. H. Kang, "Type II collagen-induced arthritis in rats. Passive transfer with serum and evidence that IgG anticollagen antibodies can cause arthritis," Journal of Experimental Medicine, vol. 155, no. 1, pp. $1-16,1982$.

[31] R. Holmdahl, M. E. Andersson, T. J. Goldschmidt et al., "Collagen induced arthritis as an experimental model for rheumatoid arthritis. Immunogenetics, pathogenesis and autoimmunity," APMIS, vol. 97, no. 7, pp. 575-584, 1989.

[32] R. O. Williams, "Rodent models of arthritis: relevance for human disease," Clinical and Experimental Immunology, vol. 114, no. 3, pp. 330-332, 1998.

[33] S. Rose, M. Eren, S. Murphy et al., "A novel mouse model that develops spontaneous arthritis and is predisposed towards atherosclerosis," Annals of the Rheumatic Diseases, vol. 72, no. 1, pp. 89-95, 2013.

[34] A. V. Misharin, G. K. Haines III, S. Rose, A. K. Gierut, R. S. Hotchkiss, and H. Perlman, "Development of a new humanized mouse model to study acute inflammatory arthritis," Journal of Translational Medicine, vol. 10, no. 1, article no. 190, 2012.

[35] A. H. Tremouler and S. Albani, "Novel therapies for rheumatoid arthritis," Expert Opinion on Investigational Drugs, vol. 15, no. 11, pp. 1427-1441, 2006.

[36] J. J. Goronzy and C. M. Weyand, "Rheumatoid arthritis," Immunological Reviews, vol. 204, no. 1, pp. 55-73, 2005.

[37] E. Gonzalez-Rey, R. Martínez-Romero, F. O’Valle et al., "Therapeutic effect of a poly(ADP-ribose) polymerase-1 inhibitor on experimental arthritis by downregulating inflammation and Th1 response," PLoS ONE, vol. 2, no. 10, Article ID e1071, 2007.

[38] R. O. Williams, "Collagen-induced arthritis in mice: a major role for tumor necrosis factor- $\alpha$," Methods in Molecular Biology, vol. 361, pp. 265-284, 2007.

[39] J. Zwerina, S. Hayer, M. Tohidast-Akrad et al., "Single and combined inhibition of tumor necrosis factor, interleukin-1, and RANKL pathways in tumor necrosis factor-induced arthritis: effects on synovial inflammation, bone erosion, and cartilage destruction," Arthritis and Rheumatism, vol. 50, no. 1, pp. 277290, 2004.

[40] K. E. Donahue, G. Gartlehner, D. E. Jonas et al., "Systematic review: comparative effectiveness and harms of diseasemodifying medications for rheumatoid arthritis," Annals of Internal Medicine, vol. 148, no. 2, pp. 124-134, 2008.

[41] L. W. Moreland, S. W. Baumgartner, M. H. Schiff et al., "Treatment of rheumatoid arthritis with a recombinant human tumor necrosis factor receptor (p75)-Fc fusion protein," The New England Journal of Medicine, vol. 337, no. 3, pp. 141-147, 1997.

[42] L. W. Moreland, M. H. Schiff, S. W. Baumgartner et al., "Phase III trial of DMARD failing rheumatoid arthritis patients with TNF receptor p75 Fc fusion protein (TNFR: Fc, ENBREL)," Journal of Investigative Medicine, vol. 46, p. 228A, 1998, Abstract.

[43] É. Toussirot and D. Wendling, "The use of TNF- $\alpha$ blocking agents in rheumatoid arthritis: an overview," Expert Opinion on Pharmacotherapy, vol. 5, no. 3, pp. 581-594, 2004.

[44] J. M. Sutherland, E. A. McLaughlin, G. R. Hime, and N. A. Siddall, "The Musashi family of RNA binding proteins: master regulators of multiple stem cell populations," Advances in Experimental Medicine and Biology, vol. 786, pp. 233-245, 2013.

[45] T. Takahashi, H. Suzuki, T. Imai et al., "Musashi-1 post-transcriptionally enhances phosphotyrosine-binding domain-containing $\mathrm{m}$-Numb protein expression in regenerating gastric mucosa," PLoS ONE, vol. 8, no. 1, Article ID e53540, 2013. 


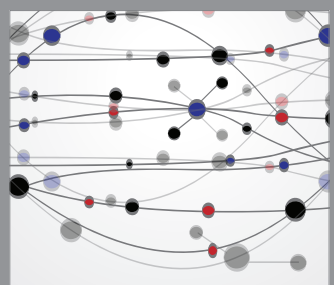

The Scientific World Journal
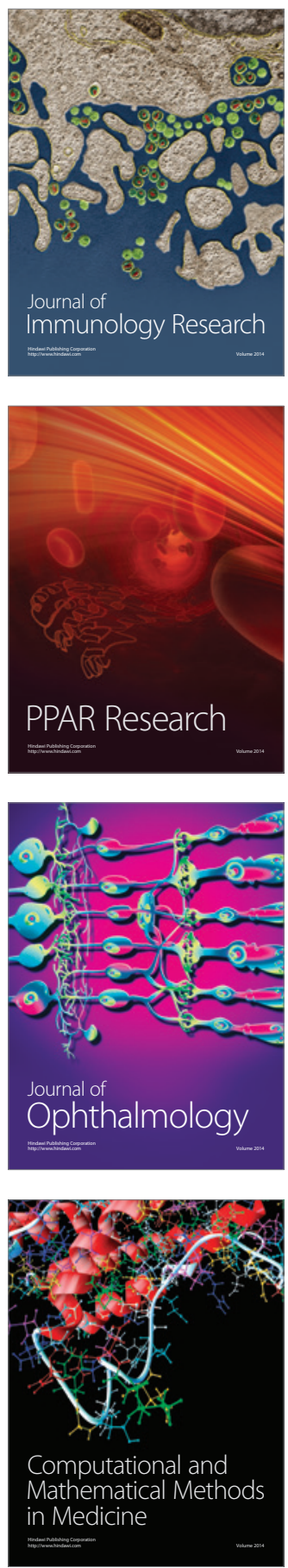

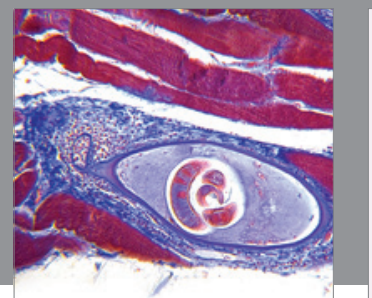

Gastroenterology

Research and Practice
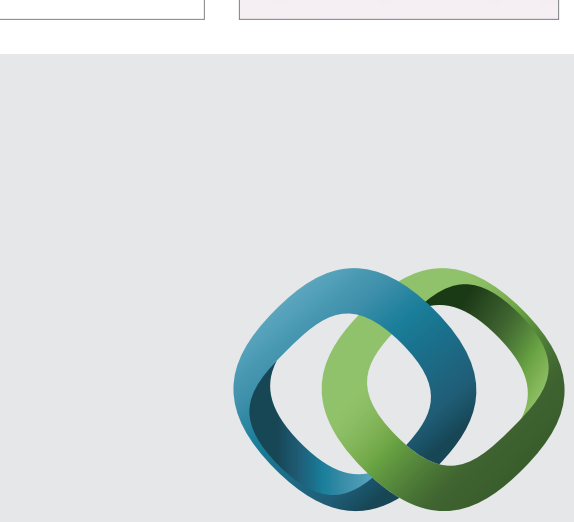

\section{Hindawi}

Submit your manuscripts at

http://www.hindawi.com
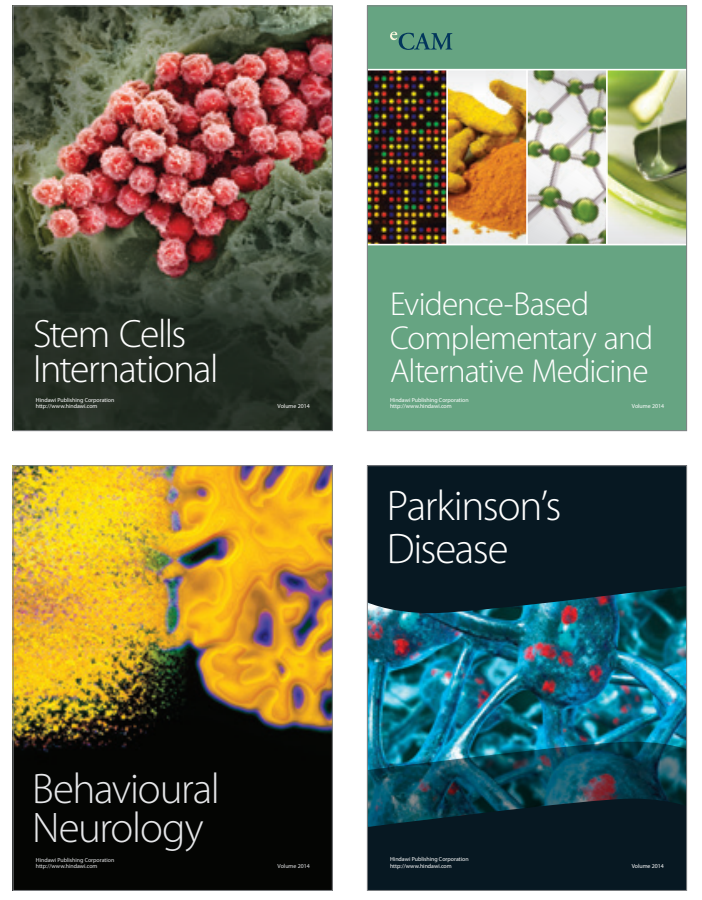
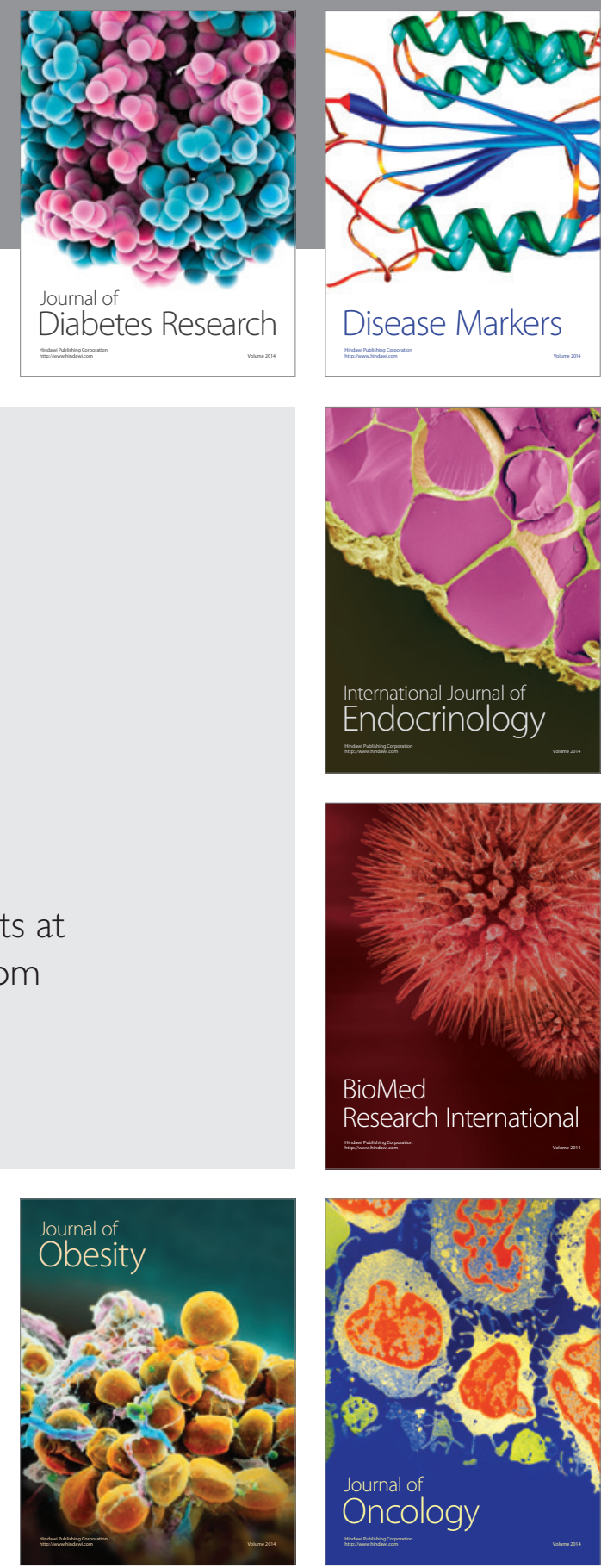

Disease Markers
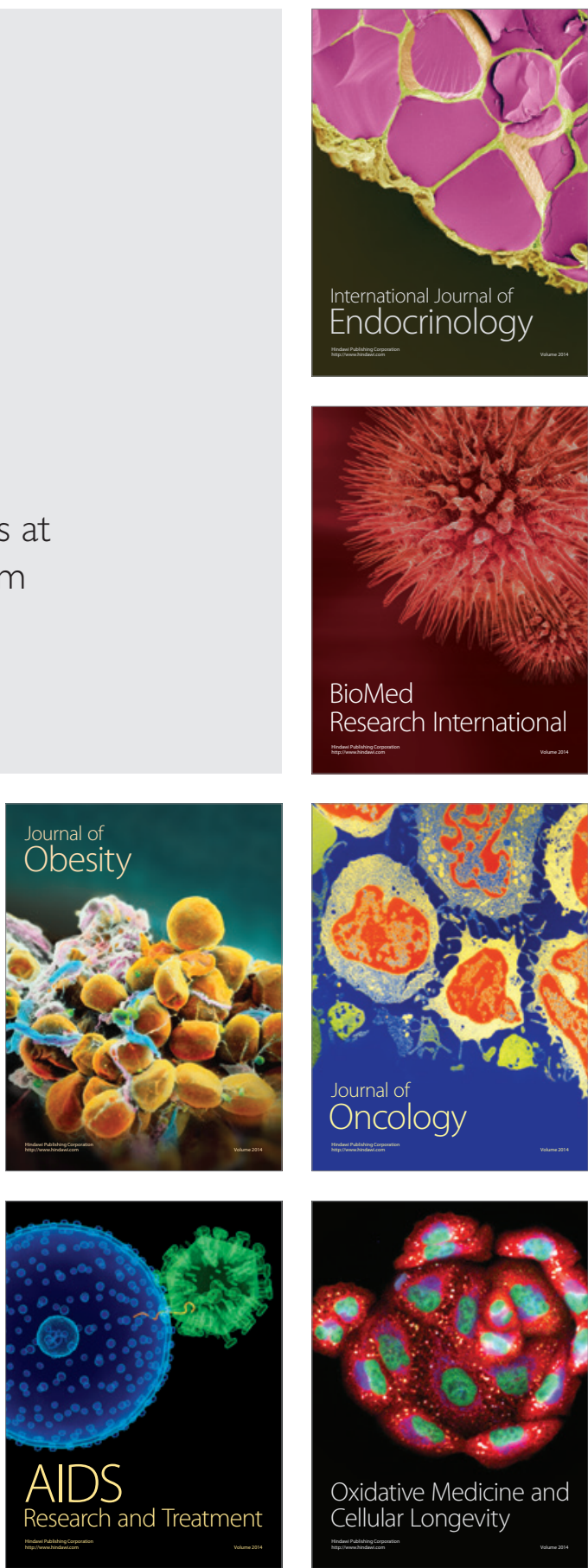